\section{PROGRAMME FOR 1971}

March 19: Heberden Round, West London Hospital and Mathilda and Terence Kennedy Institute of Rheumatology, by invitation of Dr. J. T. Scott.

June 6-11: VII European Rheumatology Congress, Brighton.

September 17-18: Clinical Meeting, Musgrave Park Hospital, Belfast.

November 26-27: The Heberden Oration (Dr. D. L. Gardner).

Annual General Meeting and Dinner. Royal College of Physicians, London.

\section{Submission of Abstracts}

The Executive Committee has decided to change the procedure by which abstracts are assessed. In future all abstracts will be considered anonymously, that is without knowledge of the name(s) of the author(s) or the institution of origin.

Members wishing to present original communications to the Society are therefore asked to prepare abstracts which provide sufficient information for assessing the paper on merit-an assessment which amounts to competitive selection between the various abstracts submitted.

In the preparation and submission of abstracts, the following points should be observed:

(1) Abstracts should not exceed 300 words. Each must be headed by a title, authors' names, institution and address.

(2) An actual summary of the communication is required. This will usually involve:

(i) A brief introduction to the work.

(ii) An outline of the methods used.

(iii) A summary of the results.

(iv) A statement of the main conclusions.

(3) Simple tables may be included. This is an excellent method of summarizing data.

(4) Accepted abstracts may be published as such with the proceedings of the Society in the Annals of the Rheumatic Diseases. So, unless they are received in a form suitable for publication, they will be returned to the author(s) for re-writing. In particular, statements such as 'The data will be discussed' are entirely unacceptable.

(5) Abstracts should be sent to the Senior Honorary Secretary, The Heberden Society, c/o Arthritis and Rheumatism Council, Faraday House, 8-10 Charing Cross Road, London, WC2H OHN. Abstracts received at least two weeks before each Executive Meeting (normally held on the same day as scientific meetings of the Society) will be assessed at that meeting.

(6) When submitting abstracts, authors must state whether the communication has been or is about to be read at another meeting; or has been or is about to be published.

(7) Authors will be notified (i) when an abstract is received, (ii) when it is either accepted or rejected, (iii) when it is placed on a programme for a particular meeting.

\section{Heberden Round}

This was conducted by Dr. J. T. Scott at the Mathilda and Terence Kennedy Institute of Rheumatology on March 19, 1971.

Dr. Scott took as his theme the problem of 'Uric Acid Ups and Downs', and showed a number of patients with various types of gout and disorders of purine metabolism.

\section{Clinical Meeting}

At a meeting held at the Kennedy Institute on March 19 , 1971, the following papers were given:

Prognosis in Systemic Sclerosis. By R. Bennett, R. Bluestone, P. J. L. Holt, and E. G. L. Bywaters (Department of Medicine, Hammersmith Hospital, London) This paper and the discussion thereon will be published in full in the Annals in November, 1971, under the title 'Survival in Scleroderma'.

Destructive Lesions of Vertebral Bodies in Ankylosing Spondylitis. By M. I. D. CAwley, T. M. Chalmers, and J. BALL (Rheumatism Research Centre, University of Manchester)

There are a number of reports in the literature of destructive lesions of the lower thoracic or lumbar vertebral bodies in patients with ankylosing spondylitis. The precise nature of these lesions is uncertain, in particular their relation to spondylitic inflammation and to non-specific factors such as trauma. In an attempt to clarify this issue, we have studied twelve patients in whom such lesions have been detected clinically and radiologically. Follow-up has varied from 0 to 16 years.

In most cases the patient described the recent onset of a pain, aggravated by exercise and relieved by rest, felt in an area consistent with the site of the lesion. Physical examination revealed local tenderness in all, with pain on jarring or forced extension of the affected area, and in some a gibbus was visible on inspection. The radiological changes varied from a localized collapse and sclerosis of the anterior part of a vertebral plate to gross destruction with collapse of two or more adjacent vertebral bodies and fracture extending to involve the neural arch.

The histological changes in cases with gross destruction are not qualitatively different from those seen in an un-united fracture. A comparison with the appearance in non-specific pseudoarthroses suggests that the inflammatory element in these spondylitic lesions may be secondary to trauma.

Treatment by immobilization of the affected area in a brace, or by surgical fixation, resulted in rapid symptomatic relief.

On clincial, radiological, and pathological grounds, it is considered that trauma is the dominant or possibly the sole aetiological factor in the production of these lesions. We emphasize that the early diagnosis of this complication depends on recognition of a qualitative and quantitative change in symptoms, and that successful management differs fundamentally from that of uncomplicated spondylitis.

\section{Discussion}

DR. G. O. STOREY (London) Your $x$ rays look like 
localized osteochondritis. Is there any similarity between these lesions and those that occur in young people without ankylosing spondylitis?

DR. CAWLEY Although we have no examples of the histology of osteochondritic lesions in non-spondylitics, Schmorl (1959) showed these lesions to consist of a herniation of the disc into the vertebral body. Radiologically and pathologically both our localized lesions and spinal osteochondritis probably represent such discal herniation.

PROF. E. G. L. BYWATERS (Hammersmith and Taplow) I agree with much that you have said about the clinical and therapeutic aspects-for instance, that these cases are best treated by immobilization. While some instances of disc collapse in ankylosing spondylitis may be triggered by trauma, in others it often occurs insidiously, and we have seen a few at autopsy. I demonstrated one of these at the Annual General Meeting of this Society in 1968; we thought then that these disc lesions were similar to the anterior discitis of Romanus lesions. If there is a traumatic element, we think that this acts on a pre-existing pathological lesion, which we think is an inflammatory discitis. In the spine of a boy with a 4-year history, $x$ rays show an inflammatory granulomatous discitis at two sites between three vertebrae which we think is similar to the inflammatory lesions that I demonstrated in 1968 in the anterior and posterior margins of the disc and also in the sacroiliac joints. The present $x$-ray slide shows depressed cartilage, obviously due to pressure, and the granulomatous disc replacement. We think that this lesion occurs in this particular position because of junctional reactions between cartilage and blood vessels at the small gaps which are sometimes seen in the basal calcified cartilage and bone layer of the epiphysis. Movement can certainly contribute to such lesions and we showed, also in 1968 , that the new bone formation responsible for the radiological appearances of the Romanus lesions, similar to the new bone formation around these disc lesions, occurs specifically at moveable segments in an otherwise immoveable spine. I do not equate movement with trauma and I do not emphasize trauma in the genesis of these lesions. I think the major factor is inflammation.

DR. D. L. GARDNER (London) You did not state at the time that the biopsies in your four cases were taken in the course of the disease. This will make a great difference in interpretation.

DR. CAWLEY I am much interested in Prof. Bywater's comments and slides. We have looked post mortem for evidence of active anterior spondylitis coexisting with destructive lesions, and have found none; nor have we found any published reports to substantiate such a relationship. We cannot claim to have proved that destructive lesions never follow an inflammatory process, but in the cases we have studied trauma seems to be dominant and we have found no evidence of inflammatory spondylitis as the initiating event.

In reply to Dr. Gardner, these patients presented several months after the change in symptoms which seems to be an important event in the evolution of these lesions, and pathological material was therefore obtained either at surgery or post mortem. To be absolutely sure of the earliest changes, one would need to have both pathological and radiological evidence at the time of the onset of the lesion and this would obviously be very difficult to obtain.

\section{Reference}

SCHMORL, G., and JUNGHANs, H. (1959) 'The Human Spine in Heal th and Disease', trans. S. P. Wilks and L. S. Goin. Grune and Stratton New York and London.

Development of Antibodies during Long-term Therapy with Tetracosactrin (Synthetic 1-24 ACTH) in Rheumatoid Arthritis. By D. Glass (Clinical Research Division, Kennedy Institute of Rheumatology and West London Hospital), G. NukI (Centre for Rheumatic Diseases and University Department of Medicine, Royal Infirmary, Glasgow), and J. R. DALY (Department of Chemical Pathology, Charing Cross Hospital Medical School)

This paper and the discussion thereon will be published in full in the Annals in November, 1971.

Cell-mediated Immunity to Micro-organisms in Rheumatoid Arthritis. By R. N. MaInI, I. T. McGrath, A. S. Russell, and D. C. Dumonde (Divisions of Clinical Research and Immunology, Kennedy Institute of Rheumatology)

Inappropriate cellular immune responses to extrinsic antigens may play a pathogenetic role in rheumatoid disease. To investigate this field, the cellular responses of lymphocyte transformation and cell migration inhibition were defined in relation to known infections (e.g. varicella, tubercle, leishmania, rubella) accompanied by clinicalo hypersensitivity states. Against this background we report the occurrence of cellular hypersensitivity to certain micro-organisms (diphtheroids, mycoplasma) that have? been associated with rheumatoid arthritis.

\section{Discussion}

DR. J. BROSTOFF (London) We have obtained different results, but I think that the two sets are not comparable in that the antigens used were different. Our antigen is sonicated mycoplasma membrane, well washed in the ultracentrifuge, and devoid of cell contents and culture medium. Yours is whole sonicated organism. It is well known that the immunological specificity of the mycoplasma rests in the membrane alone, the cytoplasm being strongly cross-reacting. It is perhaps not surprising, therefore, that you are able to show inhibition of migration in normal subjects and also a cross-reaction with M. gallisepticum.

Our own results show a good discrimination between patients with rheumatoid arthritis and those with osteoarthrosis or normal controls: in fact, the two latter groups rarely showed any inhibition. We have so far tested very few gouty subjects and the numbers are still too small for valid conclusions to be drawn. There is little evidence to support your last point that rheumatoid arthritics carry these organisms as commensals because of obvious diminution of delayed hypersensitivity; in fact, these patients give normal PHA responses and show positive skin tests to a range of antigens.

There is also overwhelming evidence for immune complexes in the joint in the form of cryoprecipitates, diminished complement level, and polymorph inclusions, so that if delayed hypersensitivity is present it may be 